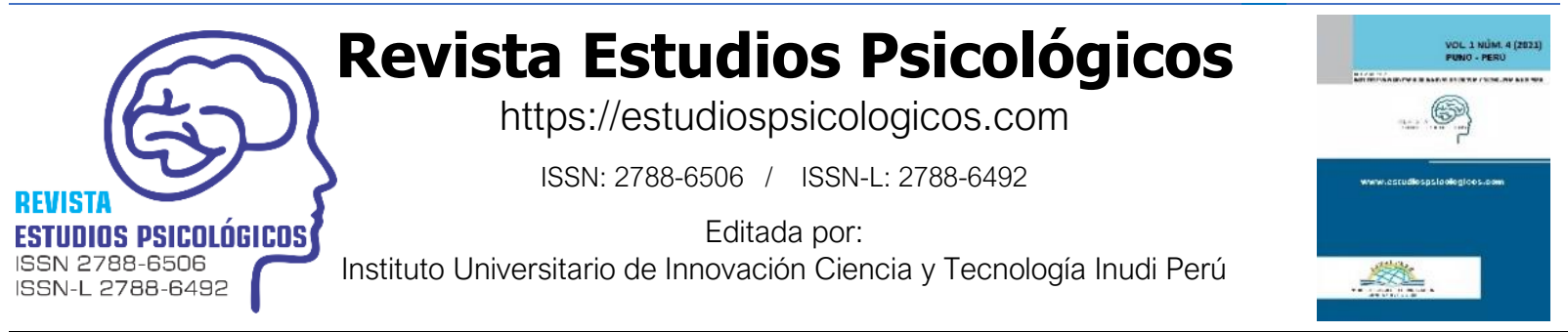

ARTÍCULO ORIGINAL

\title{
Barreras que perciben estudiantes con y sin riesgo suicida para solicitar ayuda profesional
}

\author{
Barriers perceived by students with and without suicidal risk to request professional help
}

Barreiras percebidas por alunos com e sem risco de suicídio para solicitar ajuda profissional

\author{
Daniel Paramo ${ }^{1}$ \\ Universidad de Guanajuato, León - Guanajuato, México \\ (iD) https://orcid.org/0000-0002-1792-0861
Ariel Herrera
Universidad Autónoma de San Luis Potosí, México
(iD) https://orcid.org/0000-0002-9881-3581

DOI: https://doi.org/10.35622/j.rep.2022.01.006

Recibido 24/09/2021/ Aceptado 10/01/2022

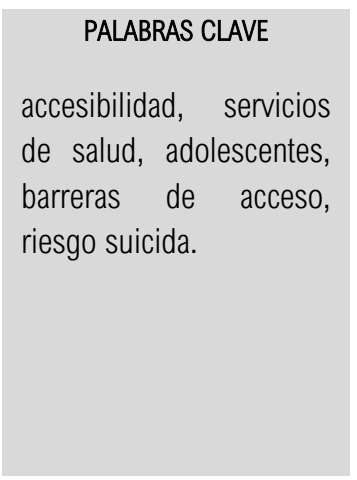

KEYWORDS

accessibility, health services, adolescents, access barriers, suicide risk.
RESUMEN. Los adolescentes tienen un alto riesgo de mortalidad por suicidio, pero su uso de servicios profesionales de salud mental es muy bajo. El objetivo fue indagar las barreras que perciben para solicitar ayuda profesional. Para esto se realizó un estudio observacional de enfoque mixto en el que se evaluó el riesgo suicida mediante la escala Plutchik. Las barreras percibidas se indagaron con una pregunta abierta que se sometió a un análisis temático de codificación abierta. Participaron 403 estudiantes de bachillerato de Aguascalientes cuyo riesgo suicida fue de 19\%. Se reconocieron 14 diferentes barreras de las que predominaron las de tipo interno: miedo y vergüenza. A mayor riesgo suicida cobraron más importancia las barreras externas como problemas familiares o las burlas. Al contrastar este con otros estudios queda de manifiesto que la perspectiva cualitativa abona una mejor comprensión de fenómenos subjetivos que subyacen a la prevención del suicidio.

ABSTRACT. Adolescents are at high risk of suicide mortality, but their use of professional mental health services is shallow. The objective was to investigate the barriers they perceive to requesting professional help. For this, an observational study with a mixed approach was carried out in which the suicide risk was evaluated using the Plutchik scale. The perceived barriers were investigated with an open question subjected to an open-coding thematic analysis. Four hundred three high school students from Aguascalientes participated, whose suicide risk was 19\%. Fourteen different barriers were recognized, of which the internal type predominated: fear and shame. At a higher risk of suicide, external walls such as family problems or teasing became more critical. When contrasting

\footnotetext{
${ }^{1}$ Correspondencia: daniel-paramo@hotmail.com
} 
this with other studies, it is clear that the qualitative perspective provides a better understanding of subjective phenomena that underlie suicide prevention.

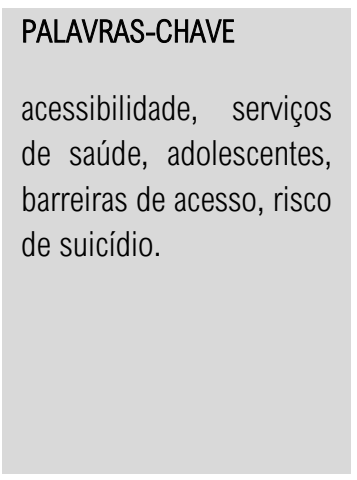

RESUM0. Os adolescentes correm alto risco de mortalidade por suicídio, mas o uso de serviços
profissionais de saúde mental é muito baixo. 0 objetivo foi investigar as barreiras que percebem
para solicitar ajuda profissional. Para isso, foi realizado um estudo observacional com abordagem
mista em que o risco de suicídio foi avaliado por meio da escala de Plutchik. As barreiras percebidas
foram investigadas com uma questão aberta que foi submetida a uma análise temática de código
aberto. Participaram 403 alunos do ensino médio de Aguascalientes, cujo risco de suicídio era de
19\%. Foram reconhecidas 14 barreiras diferentes, das quais predominou o tipo interno: medo e
vergonha. Com maior risco de suicídio, as barreiras externas, como problemas familiares ou
provocações, tornaram-se mais importantes. Ao contrastar com outros estudos, fica claro que a
perspectiva qualitativa fornece uma melhor compreensão dos fenômenos subjetivos que
fundamentam a prevenção do suicídio.

\section{INTRODUCCIÓN}

Hasta mediados de la década pasada el número de muertes por suicidio mostró un incremento sostenido (Naghavi, 2019). De modo paralelo, la prevalencia de diferentes conductas parasuicidas aumentó en gran parte del mundo (B. Han et al., 2018). Sin embargo, no hay registro de que la búsqueda de atención mental profesional por estas causas incremente también (J. Han et al., 2018).

Los jóvenes componen uno de los grupos poblacionales especialmente vulnerables para el comportamiento suicida, mismo que en algunos países como México se ubica entre las tres primeras causas de muerte entre los 15 y 19 años de edad (Borges et al., 2016; Nock et al., 2008). En nuestro país casi la mitad de los estudiantes del nivel medio superior tienen al menos un síntoma de ideación suicida (Pérez et al., 2010). Algunos de los factores de riesgo que incrementan su vulnerabilidad son: depresión, disfunción familiar, consumo de drogas, relaciones interpersonales patológicas, acoso escolar, fracaso escolar, conflictos con la propia imagen corporal o con la sexualidad y por supuesto, la presencia de intentos previos y conductas suicidas en la familia (Johns et al., 2019; Kirkcaldy et al., 2006; Picazo-Zappino, 2014; Vörös et al., 2005).

A pesar de sus factores de riesgo incrementados, es baja la utilización de servicios profesionales de salud mental por parte de los adolescentes. En Estados Unidos de Norteamérica se ha calculado que sólo 34\% de los estudiantes con un trastorno del estado de ánimo recibieron atención profesional en el último año (Blanco et al., 2008). Por su parte, quienes desarrollaron algún trastorno relacionado con sustancias el porcentaje de pacientes atendidos fue de entre el 4 y 5\% (Ebert et al., 2019; Eisenberg et al., 2007). El uso de servicios de salud mental por parte de estudiantes con ideación o riesgo suicida ronda es variado a lo largo de diferentes épocas y regiones, depende de factores personales e institucionales (Downs \& Eisenberg, 2012). Va desde el 20\% hasta el 73\% (Arria et al., 2011; Gallaguer, 2002). Se calcula que hasta un tercio de estos estudiantes rechaza los ofrecimientos de atención mental (J. Han et al., 2018). 
Hay cinco elementos que mejoran la accesibilidad de los adolescentes a los servicios de salud, ya sean públicos o privados. Se trata de: hacerlos disponibles en cantidad, cercanos, adecuados a las necesidades de los usuarios, económicamente asequibles y aceptables según las características de los servicios ofrecidos, así como de quienes brindan la atención (Garbus et al., 2017; Garduño \& Verde, 2015). Además se ha comprobado que las mujeres solicitan atención profesional en mayor medida que los varones (Nam et al., 2010). En México una Norma Oficial decretada en 2015 aspira a regular la prestación de servicios de salud para adolescentes mejorando la accesibilidad, aunque sin un estudio previo de las barreras, reales o percibidas, para hacer uso de tales servicios.

Diversos estudios de las barreras de los estudiantes para no acudir con un profesional se encontraron que la principal fue que se minimiza el riesgo y por tanto consideran que su malestar no merece atención profesional por ser común o transitorio, esto en $66.2 \%$ de las ocasiones. Le siguieron la falta de falta de tiempo con $26 \%$ y preferencia por manejar ellos mismos su problema con 17.8\% (Czyz et al., 2013). Además, se sabe que la mayoría de los adolescentes acuden en primer lugar con sus padres cuando padecen algún malestar físico 0 mental (Garduño \& Verde, 2015). Esto ocurre con más frecuencia cuando: los adolescentes tienen una actitud positiva hacia pedir ayuda, cuando creen que les responderán o se preocuparán, que cuentan con confidencialidad y además si mantienen apego a la escuela (Pisani et al., 2013).

Negarse a recibir ayuda profesional es un fenómeno comúnmente asociado al comportamiento suicida (Barnes et al., 2002; Rudd et al., 1995). Esta negación puede estar basada en la percepción de barreras para el acceso a servicios profesionales de salud. Y sin embargo, entender las barreras para atender el comportamiento suicida es un elemento clave en la prevención del suicidio en escolares (Balaguru et al., 2013). Por tanto, el objetivo de esta investigación fue identificar las barreras que perciben los estudiantes para buscar atención profesional en caso de riesgo suicida.

\section{METODO Y MATERIALES}

El presente es un estudio descriptivo de tipo observacional, retrospectivo y transversal. El enfoque fue mixto: se realizó un análisis cualitativo de preguntas abiertas y en muchas ocasiones polisémicas (Rodríguez et al., 2005). Posteriormente se realizó un análisis cuantitativo de estas categorías previamente construidas, así como de las otras variables.

Los instrumentos de recolección de la información fueron el cuestionario Plutchik de evaluación del riesgo suicida y una pregunta abierta acerca de por qué creen que los estudiantes con riesgo suicida no solicitan ayuda 
profesional. Se planteó así en tercera persona y sin más indicaciones ni límite de respuestas o de extensión para fomentar la expresión libre de censuras personales. El test Plutchik por su parte es una escala que evalúa el riesgo suicida mediante 15 ítems de respuesta dicotómica. Su versión en español fue validada por Suárez et al. (2019) quienes obtuvieron una confiabilidad de $\alpha=0.80$. El punto de corte para determinar el riesgo elevado es de 6 puntos.

Participaron 403 estudiantes de un bachillerato público de la capital de Aguascalientes. El procedimiento seguido fue aplicarle los instrumentos a los casi dos mil alumnos del plantel como parte de un proyecto más amplio en varias unidades académicas de Aguascalientes. De la base de datos general se seleccionaron aleatoriamente con la función propia del programa SPSS al 25\% de los participantes. De estos se descartaron 83 casos por diversas razones: no consignaron sus datos personales, no contestaron completamente a los instrumentos o no respondieron a la pregunta abierta. Se contó con el consentimiento informado de los alumnos, además de que la participación en la investigación fue voluntaria y confidencial.

El análisis de la información se llevó a cabo con dos programas computacionales diferentes. Para el cálculo del riesgo suicida y el análisis de las variables psicosociales se utilizó el SPSS (v.19) y se analizó mediante estadísticos descriptivos y la prueba ANOVA. Las respuestas abiertas una vez codificadas se analizaron con la versión de Anthopac 4.98 para Windows a través de porcentajes e índice S de Smith, mismo que considera la cantidad de menciones y el lugar en que se refiere cada concepto en el discurso individual. El procedimiento para codificar las preguntas abiertas fue llevado a cabo por un equipo de psicólogos tomando como unidades de contenido un criterio temático, es decir, por los elementos a que se referían los estudiantes en su respuesta (Rodríguez et al., 2005). Las categorías fueron construidas con base en las mismas respuestas en una codificación dinámica (Ryan \& Rusell, 2000).

\section{RESULTADOS Y DISCUSIONES}

De los 403 estudiantes 255 (56\%) fueron mujeres y 178 (44\%) hombres; 158 pertenecían al segundo semestre (39\%), 110 de cuarto (27\%) y 135 del sexto (33\%). La edad de los estudiantes fue de los 14 a los 19 años con una media de 16. La mayoría no trabajan, 25\% lo hacen ocasionalmente y sólo 9\% permanentemente. En ninguna de estas variables hubo diferencias significativas por género o según el grado de riesgo suicida hallado.

Se encontró elevado riesgo suicida en 19.4\% de los estudiantes. De los 15 puntos posibles en el test Plutchik el rango de respuestas fue de 0 a un máximo de 14, con promedio de 3.22 y desviación estándar de 2.9. De 
los tres semestres analizados fue ligeramente mayor entre los de segundo (3.4) y menor en los de sexto (2.9), sin que las diferencias sean significativas ( $F=1.05, \mathrm{p}=0.350)$.

Se categorizaron 14 diferentes razones por las que un estudiante con riesgo suicida no pide ayuda profesional. En promedio cada participante dijo 1.27 conceptos. Predominaron vergüenza y miedo con 31 y $30 \%$ de las menciones cada uno. Le siguió Falta de conocimiento con 14\% de alumnos mencionándola; este concepto incluyó estigmas sobre la salud mental (porque son personas cerradas, caso 138; sólo quieren estar aislados de los demás, caso 53), y creencias erróneas sobre su tratamiento (que no va a servir, caso 267; que los van a tratar como si estuvieran locos, caso 224). Desconfianza, que fue el quinto lugar, se refirió al escepticismo hacia los tratamientos. El resto de las barreras para buscar ayuda profesional se refieren en la Figura 1.

Figura 1. Barreras para la atención profesional percibidas por los estudiantes en general y por los que tienen riesgo suicida.

\begin{tabular}{|c|c|c|c|c|c|c|c|c|}
\hline \multicolumn{3}{|c|}{$\begin{array}{l}\text { ESTUDIANTES EN GENERAL (Plutchik =3.22) } \\
\qquad(\mathrm{N}=403)\end{array}$} & \multicolumn{3}{|c|}{$\begin{array}{l}\text { CON RIESGO SUICIDA (Plutchik } \geq 6 \text { ) } \\
\qquad(n=78)\end{array}$} & \multicolumn{3}{|c|}{$\begin{array}{l}\text { MUY ALTO RIESGO SUICIDA (Plutchik } \geq 8 \\
\qquad(n=41)\end{array}$} \\
\hline$\%$ & Smith's S & BARRERA & BARRERA & $\%$ & Smith's S & BARRERA & $\%$ & Smith's S \\
\hline 31.4 & 0.28 & Vergüenza & Vergüenza & 29.9 & 0.27 & Vergüenza & 32.5 & 0.32 \\
\hline 30.7 & 0.29 & Miedo & Miedo & 27.3 & 0.26 & Miedo & 32.5 & 0.31 \\
\hline 13.9 & 0.19 & Falta de conocimiento & Falta de conocimiento & 16.9 & 0.13 & Problemas familiares & 20 & 0.16 \\
\hline 11.9 & 0.11 & Falta de dinero & Problemas familiares & 14.3 & 0.12 & Falta de conocimiento & 17.5 & 0.10 \\
\hline 10.4 & 0.09 & Desconfianza & Falta de dinero & 13 & 0.11 & Falta de dinero & 12.5 & 0.10 \\
\hline 9.2 & 0.08 & Problemas familiares & Desconfianza & 9.1 & 0.09 & Burlas & 7.5 & 0.05 \\
\hline 7.7 & 0.06 & Desinterés & Burlas & 5.2 & 0.03 & Falta de tiempo & 5 & 0.03 \\
\hline 5 & 0.03 & Burlas & Desinterés & 3.9 & 0.04 & Desinterés & 5 & 0.05 \\
\hline 1.7 & 0.01 & Falta de tiempo & Falta de tiempo & 2.6 & 0.02 & Falta de aceptación & 2.5 & 0.02 \\
\hline 1.5 & 0.01 & Incomodidad & Falta de apoyo & 2.6 & 0.01 & Incomodidad & 2.5 & 0.02 \\
\hline 1.2 & 0.01 & Falta de aceptación & \begin{tabular}{|l} 
Flojera \\
\end{tabular} & 2.6 & 0.02 & Falta de apoyo & 2.5 & $<0.01$ \\
\hline 1 & $<0.01$ & Falta de apoyo & Falta de aceptación & 1.3 & 0.01 & & & \\
\hline 0.7 & $<0.01$ & Flojera & Incomodidad & 1.3 & 0.01 & & & \\
\hline 0.7 & $<0.01$ & Contexto & & & & & & \\
\hline
\end{tabular}

Fuente: elaboración propia.

Al clasificar las barreras percibidas en externas e internas predominó en todos los estudiantes las de tipo interno, señaladas en azul en la figura, tales como la vergüenza, miedo y hasta flojera. En el otro extremo y en color rojo se ubicaron barreras externas como problemas familiares, burlas y bullying o falta de apoyo. La falta de dinero y de tiempo no se catalogaron en ninguno de estos conjuntos porque dependen de diversas circunstancias. Destacan las barreras personales como la vergüenza y el miedo, pero a mayor riesgo suicida más importancia van cobrando las barreras externas como la presencia de problemas familiares o burlas por pedir ayuda.

En un segundo análisis se compararon las barreras percibidas por los estudiantes en general con aquellas mencionadas por los que tienen alto y muy alto riesgo suicida porque obtuvieron más de 6 y de 8 puntos en la escala Plutchik, respectivamente. Esta comparación también se muestra en la Figura 1. Destaca que a mayor riesgo suicida se percibían como más importantes las barreras externas. Quienes no tienen riesgo mencionaron 
$78 \%$ de conceptos de índole interna y $11 \%$ son del entorno, mientras que aquellos con muy elevado riesgo suicida consideran que $66 \%$ de los obstáculos son personales y $21 \%$ son de su entorno.

\section{DISCUSIÓN}

La aproximación cualitativa permitió identificar un gran número de barreras para la atención a la salud percibidas por los estudiantes en comparación con estudios que las codifican y prevén con anticipación (Czyz et al., 2013). Adicionalmente se consiguió recuperar un amplio universo semántico de expresiones, ejemplos 0 connotaciones que los participantes le dan a estas barreras. La falta de conocimiento, por ejemplo, hizo alusión al desconocimiento tanto de servicios profesionales disponibles, como a las formas de acceder a ellos y de los factores de riesgo para el suicidio. Las intervenciones que abordan esta falta de conocimiento son denominadas alfabetización en salud, y son reconocidas como importantes dianas para la prevención, no solamente para atender las conductas suicidas sino la salud mental en general (Aguirre et al., 2020; Calear et al., 2014; Chan et al., 2014; Kim et al., 2020).

Otro dato revela la poca alfabetización en salud en este tema tan íntimo como lo es la percepción de barreras y el eventual uso de servicios de salud mental. En promedio cada alumno refirió 1.2 barreras. Se considera que al ser un tema difícil de identificar y muchas veces ajeno a la experiencia de los estudiantes, las técnicas cualitativas tienen mejor capacidad exploratoria.

Aunque los estudiantes les otorgaron más peso a los factores intrapersonales, se observa el importante papel que tienen los procesos de estigmatización sobre quien tiene un padecimiento emocional, incluyendo el acoso escolar. No obstante, en el proceso de codificación de las barreras no se utilizó la categoría estigmas por su compleja naturaleza tanto social como internalizada (Eisenberg et al., 2009). Donde sus efectos van más allá de dificultar el acceso a los servicios de salud, hasta llegar incluso a acelerar el proceso suicida y agravar otros factores de riesgo (0exle et al., 2020).

Tres de las barreras aquí identificadas conciernen directamente al sistema de salud y se ubican entre las cinco más importantes: falta de conocimiento, de dinero y desconfianza. Estos temas son abordados en la Norma Oficial Mexicana (NOM-047-SSA2-2015 Para la atención a la salud del Grupo Etario de 10 a 19 años de edad, 2019) y en guías de servicios de salud amigables para los adolescentes. Es por esto que estudios como el que aquí se presentan aportan información para realizar intervenciones preventivas individualizadas para cada población (Hunt \& Eisenberg, 2010; Sagar et al., 2020). Porque no sólo los servicios de salud son diferentes entre regiones, sino que también lo son las barreras percibidas, si existen condiciones de salud agravantes (Eisenberg et al., 2011; Fox et al., 2020). 
El riesgo suicida de $19 \%$ de los estudiantes fue muy similar al encontrado en otros bachilleratos del mismo estado de Aguascalientes (Herrera et al., 2015). En la República Mexicana se han encontrado prevalencias de riesgo suicida en hasta $47 \%$ de en poblaciones similares (Pérez et al., 2010). Desafortunadamente las metodologías e instrumentos utilizados en estos estudios dificultan la comparación directa de resultados.

Se considera que estudiar es un factor protector de intentos suicidas en la adolescencia (Mortier et al., 2018). Pero la búsqueda de atención a la salud no es mayor entre estudiantes y población abierta, o entre estudiantes a tiempo completo o de tiempo parcial (B. Han et al., 2016).

\section{CONCLUSIONES}

Se identificaron 14 diferentes barreras para la atención a la salud mental para atención del riesgo suicida. La pregunta abierta e indirecta permitió la expresión de distintas ideas y circunstancias que ponen de relieve la complejidad de este tema pues se reconocen factores personales, del entorno familiar, el social y de la misma provisión de servicios de salud.

Otro hallazgo de relevancia es que los estudiantes que presentan más alto riesgo suicida le atribuyen más importancia a las barreras externas en comparación con quienes tienen bajo riesgo. Son, en otras palabras, más sensibles a la ausencia de apoyo familiar, a las burlas y falta de dinero y de tiempo. Este estudio da una imagen de las circunstancias del estudiantado y particularmente de quienes tienen riesgo suicida, al tiempo que da pautas para un abordaje oportuno y preciso.

\section{Conflicto de intereses / Competing interests:}

Los autores declaran que no incurre en conflictos de intereses.

\section{Rol de los autores / Authors Roles:}

Daniel Paramo: conceptualización, curación de datos, análisis formal, adquisición de fondos, investigación, metodología, administración del proyecto, recursos, software, supervisión, validación, visualización, escritura preparación del borrador original, escritura - revisar \& amp; edición.

Ariel Herrera: conceptualización, análisis formal, investigación, metodología, administración del proyecto, recursos, software, supervisión, validación, visualización, escritura - preparación del borrador original, escritura - revisar \& amp; edición.

\section{Fuentes de financiamiento / Funding:}

Los autores declaran que no recibió un fondo específico para esta investigación.

\section{Aspectos éticos / legales; Ethics / legals:}

Los autores declaran no haber incurrido en aspectos antiéticos, ni haber omitido aspectos legales en la realización de la investigación.

\section{REFERENCIAS}

Aguirre, A., Cruz, S., Billings, J., Jimenez, M., \& Rowe, S. (2020). What are the barriers, facilitators and interventions targeting help-seeking behaviours for common mental health problems in adolescents? A systematic review. BMC Psychiatry, 20(1), 293. https://doi.org/10.1186/s12888-020-02659-0 
Arria, A. M., Winick, E. R., Garnier-Dykstra, L. M., Vincent, K. B., Caldeira, K. M., Wilcox, H. C., \& O'Grady, K. E. (2011). Help Seeking and Mental Health Service Utilization Among College Students With a History of Suicide Ideation. Psychiatric Services, 62(12), 1510-1513. https://doi.org/10.1176/appi.ps.005562010

Balaguru, V., Sharma, J., \& Waheed, W. (2013). Understanding the effectiveness of school-based interventions to prevent suicide: a realist review. Child and Adolescent Mental Health, 18(3), 131-139. https://doi.org/10.1111/j.1475-3588.2012.00668.x

Barnes, L. S., Ikeda, R. M., \& Kresnow, M. (2002). Help-Seeking Behavior Prior to Nearly Lethal Suicide Attempts. Suicide and Life-Threatening Behavior, 32, 68-75. https://doi.org/10.1521/suli.32.1.5.68.24217

Blanco, C., Okuda, M., Wright, C., Hasin, D. S., Grant, B. F., Liu, S.-M., \& Olfson, M. (2008). Mental Health of College Students and Their Non-College-Attending Peers. Archives of General Psychiatry, 65(12), 1429. https://doi.org/10.1001/archpsyc.65.12.1429

Borges, G., García, J., \& Borsani, L. (2016). Epidemiología de las conductas suicidas en México. En OPS (Ed.), Prevención de la conducta suicida (1. ${ }^{\text {a }}$ ed., pp. 48-56). OPS.

Calear, A. L., Batterham, P. J., \& Christensen, H. (2014). Predictors of help-seeking for suicidal ideation in the community: Risks and opportunities for public suicide prevention campaigns. Psychiatry Research, 219(3), 525-530. https://doi.org/10.1016/j.psychres.2014.06.027

NOM-047-SSA2-2015 Para la atención a la salud del Grupo Etario de 10 a 19 años de edad, (2019).

Chan, W. I., Batterham, P., Christensen, H., \& Galletly, C. (2014). Suicide literacy, suicide stigma and helpseeking intentions in Australian medical students. Australasian Psychiatry, 22(2), 132-139. https://doi.org/10.1177/1039856214522528

Czyz, E. K., Horwitz, A. G., Eisenberg, D., Kramer, A., \& King, C. A. (2013). Self-reported Barriers to Professional Help Seeking Among College Students at Elevated Risk for Suicide. Journal of American College Health, 61(7), 398-406. https://doi.org/10.1080/07448481.2013.820731

Downs, M. F., \& Eisenberg, D. (2012). Help Seeking and Treatment Use Among Suicidal College Students. Journal of American College Health, 60(2), 104-114. https://doi.org/10.1080/07448481.2011.619611

Ebert, D. D., Mortier, P., Kaehlke, F., Bruffaerts, R., Baumeister, H., Auerbach, R. P., Alonso, J., Vilagut, G., Martínez, K. U., Lochner, C., Cuijpers, P., Kuechler, A., Green, J., Hasking, P., Lapsley, C., Sampson, N. A., \& Kessler, R. C. (2019). Barriers of mental health treatment utilization among first-year college students: First cross-national results from the WHO World Mental Health International College Student Initiative. 
International Journal of Methods in Psychiatric Research, 28(2), e1782. https://doi.org/10.1002/mpr.1782

Eisenberg, D., Downs, M. F., Golberstein, E., \& Zivin, K. (2009). Stigma and Help Seeking for Mental Health Among College Students. Medical Care Research and Review, 66(5), 522-541. https://doi.org/10.1177/1077558709335173

Eisenberg, D., Golberstein, E., \& Gollust, S. E. (2007). Help-Seeking and Access to Mental Health Care in a University Student Population. Medical Care, 45(7), 594-601. https://doi.org/10.1097/MLR.0b013e31803bb4c1

Eisenberg, D., Hunt, J., Speer, N., \& Zivin, K. (2011). Mental Health Service Utilization Among College Students in the United States. Journal of Nervous \& Mental Disease, 199(5), 301-308. https://doi.org/10.1097/NMD.0b013e3182175123

Fox, M. L., James, T. G., \& Barnett, S. L. (2020). Suicidal Behaviors and Help-Seeking Attitudes Among Deaf and Hard-of-Hearing College Students. Suicide and Life-Threatening Behavior, 50(2), 387-396. https://doi.org/10.1111/sltb.12595

Garbus, P., Morales Osornio, A., \& Cabello Álvarez, L. S. (2017). Accesibilidad a la atención en salud de adolescentes en San Juan del Río, Querétaro (México). Enseñanza e Investigación en Psicología, 22(3), 277-285.

Garduño, F., \& Verde, J. (2015). La dimensión simbólica de la accesibilidad a la atención en salud de los adolescentes de San Juan del Río, Querétaro, México. Universidad Autónoma de Querétaro.

Han, B., Compton, W. M., Blanco, C., Colpe, L., Huang, L., \& McKeon, R. (2018). National Trends in the Prevalence of Suicidal Ideation and Behavior Among Young Adults and Receipt of Mental Health Care Among Suicidal Young Adults. Journal of the American Academy of Child \& Adolescent Psychiatry, 57(1), 20-27.e2. https://doi.org/10.1016/j.jaac.2017.10.013

Han, B., Compton, W. M., Eisenberg, D., Milazzo-Sayre, L., McKeon, R., \& Hughes, A. (2016). Prevalence and Mental Health Treatment of Suicidal Ideation and Behavior Among College Students Aged 18-25 Years and Their Non-College-Attending Peers in the United States. The Journal of Clinical Psychiatry, 77(06), 815-824. https://doi.org/10.4088/JCP.15m09929

Han, J., Batterham, P. J., Calear, A. L., \& Ma, J. (2018). Seeking professional help for suicidal ideation: A comparison between Chinese and Australian university students. Psychiatry Research, 270, 807-814. https://doi.org/10.1016/.jpsychres.2018.10.080 
Herrera, A., Páramo, D., \& de Alba, D. (2015). Relación entre calidad de las relaciones intrafamiliares y riesgo de suicidio en estudiantes de Aguascalientes. VI Congreso Internacional de Prevención del Suicidio, 191199.

Hunt, J., \& Eisenberg, D. (2010). Mental Health Problems and Help-Seeking Behavior Among College Students. Journal of Adolescent Health, 46(1), 3-10. https://doi.org/10.1016/j.jadohealth.2009.08.008

Johns, M. M., Lowry, R., Andrzejewski, J., Barrios, L. C., Demissie, Z., McManus, T., Rasberry, C. N., Robin, L., \& Underwood, J. M. (2019). Transgender Identity and Experiences of Violence Victimization, Substance Use, Suicide Risk, and Sexual Risk Behaviors Among High School Students — 19 States and Large Urban School Districts, 2017. MMWR. Morbidity and Mortality Weekly Report, 68(3), 67-71. https://doi.org/10.15585/mmwr.mm6803a3

Kim, E. J., Yu, J. H., \& Kim, E. Y. (2020). Pathways linking mental health literacy to professional help-seeking intentions in Korean college students. Journal of Psychiatric and Mental Health Nursing, 27(4), 393-405. https://doi.org/10.1111/jpm.12593

Kirkcaldy, B. D., Siefen, G. R., Urkin, J., \& Merrick, J. (2006). Risk factors for suicidal behavior in adolescents. Minerva pediatrica, 58(5), 443-450.

Mortier, P., Auerbach, R. P., Alonso, J., Axinn, W. G., Cuijpers, P., Ebert, D. D., Green, J. G., Hwang, I., Kessler, R. C., Liu, H., Nock, M. K., Pinder-Amaker, S., Sampson, N. A., Zaslavsky, A. M., Abdulmalik, J., AguilarGaxiola, S., Al-Hamzawi, A., Benjet, C., Demyttenaere, K., ... Bruffaerts, R. (2018). Suicidal thoughts and behaviors among college students and same-aged peers: results from the World Health Organization World Mental Health Surveys. Social Psychiatry and Psychiatric Epidemiology, 53(3), 279-288. https://doi.org/10.1007/s00127-018-1481-6

Naghavi, M. (2019). Global, regional, and national burden of suicide mortality 1990 to 2016: systematic analysis for the Global Burden of Disease Study 2016. BMJ, 194. https://doi.org/10.1136/bmj.I94

Nam, S. K., Chu, H. J., Lee, M. K., Lee, J. H., Kim, N., \& Lee, S. M. (2010). A Meta-analysis of Gender Differences in Attitudes Toward Seeking Professional Psychological Help. Journal of American College Health, 59(2), 110-116. https://doi.org/10.1080/07448481.2010.483714

Nock, M. K., Borges, G., Bromet, E. J., Cha, C. B., Kessler, R. C., \& Lee, S. (2008). Suicide and Suicidal Behavior. Epidemiologic Reviews, 30(1), 133-154. https://doi.org/10.1093/epirev/mxn002

Oexle, N., Mayer, L., \& Rüsch, N. (2020). Suizidstigma und Suizidprävention. Der Nervenarzt, 91(9), 779-784. 
https://doi.org/10.1007/s00115-020-00961-6

Pérez, B., Rivera, L., Atienzo, E., Castro, F., Leyva, A., \& Chávez, R. (2010). Prevalencia y factores asociados a la ideación e intento suicida en adolescentes de educación media superior de la República Mexicana. Salud Pública de México, 52, 324-333. http://disde.minedu.gob.pe/handle/20.500.12799/1288

Picazo-Zappino, J. (2014). Suicide among children and adolescents: a review. Actas espanolas de psiquiatria, $42(3), 125-132$.

Pisani, A. R., Wyman, P. A., Petrova, M., Schmeelk-Cone, K., Goldston, D. B., Xia, Y., \& Gould, M. S. (2013). Emotion Regulation Difficulties, Youth-Adult Relationships, and Suicide Attempts Among High School Students in Underserved Communities. Journal of Youth and Adolescence, 42(6), 807-820. https://doi.org/10.1007/s10964-012-9884-2

Rodríguez, C., Lorenzo, 0., \& Herrera, L. (2005). Teoría y práctica del análisis de datos cualitativos. Proceso general y criterios de calidad. Revista Internacional de Ciencias Sociales y Humanidades, SOCIOTAM, XV(2), 133-154. https://www.redalyc.org/articulo.oa?id=65415209

Rudd, M. D., Joiner, T. E., \& Rajab, M. H. (1995). Help negation after acute suicidal crisis. Journal of Consulting and Clinical Psychology, 63(3), 499-503. https://doi.org/10.1037/0022-006X.63.3.499

Ryan, G., \& Rusell, B. (2000). Data management and analysis methods. En N. Denzing \& I. Lincoln (Eds.), Handbook of qualitative research (2. ${ }^{a}$ ed., pp. 769-802). SAGE.

Sagar, I., Godfrey, E., Graham, S., \& Brown, J. S. L. (2020). Improving Mental Health Help-Seeking Behaviours for Male Students: A Framework for Developing a Complex Intervention. International Journal of Environmental Research and Public Health, 17(14), 4965. https://doi.org/10.3390/ijerph17144965

Suárez, Y., Palacio, J., Caballero, C., \& Pineda, C. (2019). Adaptación, validez de constructo y confiabilidad de la escala de riesgo suicida Plutchik en adolescentes colombianos. Revista Latinoamericana de Psicología, 51(3). https://doi.org/10.14349/rlp.2019.v51.n3.1

Vörös, V., Fekete, S., Hewitt, A., \& Osváth, P. (2005). [Suicidal behavior in adolescents--psychopathology and addictive comorbidity]. Neuropsychopharmacologia Hungarica : a Magyar Pszichofarmakologiai Egyesulet lapja = official journal of the Hungarian Association of Psychopharmacology, 7(2), 66-71. 